\title{
A method for research viscoplastic characteristics of materials using a vertical gas-gun stand
}

\author{
V.G. Bazhenov ${ }^{1}$, M.S. Baranova², and E.V. Pavlyonkova ${ }^{2}$ \\ ${ }^{1}$ Researh Institute of Mechanics Lobachevsky State University of Nizhniy Novgorod, \\ pr. Gagarina 23/6 Nizhniy Novgorod, Russia \\ 2 Lobachevsky State University of Nizhniy Novgorod, pr. Gagarina 23 Nizhniy Novgorod, Russia
}

\begin{abstract}
A methodology of constructing dynamic strain diagrams based on the method of direct impact on a vertical gas-gun stand has been developed and theoretically verified. The methodology has been verified by a reconstruction of a given static strain diagram when substituting the physical experiment by computer modeling of an axisymmetric problem, accounting for the wave processes in the striker-specimen-measuring rod system. The main inaccuracies in determining (reconstructing) viscoplastic characteristics of materials are shown to be determined by the degree of accuracy of experimental measurements. Based on the method of characteristics, an algorithm has been developed for calculating the displacements of the impacted end of the rod based on the results of the measurements of strains by two gauges in the vicinity of the impacted and supported ends of the rod for a multiply passing strain wave. This makes it possible to analyze the deformation process up to the moment the striker stops and to compare the computational and experimental results based on the residual size of the tested specimen.
\end{abstract}

\section{Introduction}

The impact direct method is used for research elastoviscoplastic properties of materials in range of strain rates $10^{1}-10^{4} 1 / \mathrm{s}[1-3]$. One of the experimental stand realizing this method is vertical gas-gun stand.

Vertical gas-gun stand (figure 1) contains the following elements: striker (marked 1), specimen (2), measuring $\operatorname{rod}(3)$. Gas-gun stand supports on a laminose base.

Stress in specimen is defined based on strain pulse which is registered in the measuring rod. For calculation of strain in the specimen there are assumptions about striker and specimen. Striker is undeformed. Material of specimen is incompressible.

\section{One-dimensional model of the wave process in the striker-specimen-measuring rod system}

An initial velocity of striker is registered in the experiment. A strain gauge which is located on the measuring rod at location $\mathrm{A}$ at the distance $l>=2 \mathrm{R}$ registers strain pulse at the end of the rod with time-shift equaling time of compressional wave propagation along specimen and the measuring rod from location of impact to stain gauge location. There are the following assumptions:

1) Transverse oscillation of rods is not considered.

2) Striker is absolutely hard.

3) Stress-strain state is considered homogeneous because of size of specimen is small.

Axial force $F_{z}^{A}$ located in the elastic measuring rod at location A is defined by Hooke's law

$$
F_{z}^{A}=\pi R_{1}^{2} \sigma_{z}^{A}, \quad \sigma_{z}^{A}=E e_{z}^{A}
$$

where $E$ is Young's modulus in the measuring $\operatorname{rod}, \sigma_{z}^{A}$ - is stress in the measuring rod at location A.
Length change $L_{2}$ of the specimen can be written knowing displacement of the contact surfaces of the striker $u_{z}^{1}$ and the measuring $\operatorname{rod} u_{z}^{3}$ :

$$
L_{2}=L_{20}-\left(u_{z}^{3}-u_{z}^{1}\right)
$$

where $L_{20}$ is the initial length of the specimen.

Displacement of the contact surface of the measuring rod $u_{z}^{3}$ can be written through elastic strain which is registered in the measuring $\operatorname{rod} e_{z}^{A}$

$$
u_{z}^{3}=c \int_{0}^{t} e_{z}^{A}(t) d t
$$

where $c=\sqrt{E / \rho}$ is a velocity of elastic wave in the measuring rod.

The displacement of the contact surface of the striker $u_{z}^{1}$ can be found using integration of equation of motion with the desired initial conditions:

$$
m^{1} \ddot{u}_{z}^{1}=F_{z}^{A},\left.\quad \dot{u}_{z}^{1}\right|_{t=0}=V_{0},\left.\quad u_{z}^{1}\right|_{t=0}=0
$$

In the right part of the equation of motion $F_{z}^{A}$ is an axial force which is registered in the experiment in the measuring rod, $m^{1}$ - a mass of the striker.

Using the length, which is defined earlier, nominal and true axis strains and strain rate in the specimen are defined

$$
\bar{e}_{z}=\frac{L_{2}-L_{20}}{L_{20}}, \quad e_{z}=\ln \frac{L_{2}}{L_{20}}=\ln \left(1+\bar{e}_{z}\right), \quad \dot{e}_{z}=\frac{d e_{z}}{d t}
$$

The axis stress is calculated based on incompressible condition using changing of cross-section

$$
\sigma_{z}=\frac{F_{z}^{A}\left(1-\bar{e}_{z}\right)}{S_{20}}
$$

where $S_{20}=\pi R_{20}^{2}$ and $R_{20}$ are a square and a radius of undeformed specimen correspondingly. 


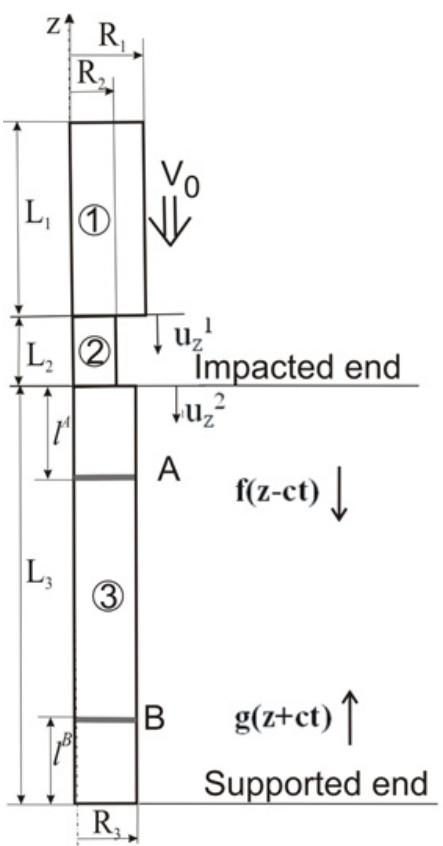

Fig. 1. Gas-gun stand scheme.

Thus, using experimental data such as dependence of axis stress in time $F_{z}^{A} \sim t$ in the measuring rod and the initial velocity $V_{0}$ of the striker the dependence of axis stress- axis strain $\sigma_{z} \sim e_{z}$ in the specimen is built in the range of rate strains which are realized in the experiment. Verification of this methodology is presented in [4].

\section{A methodology of wave process reconstruction in the measuring rod based on two strain gauges}

The methodology of building the stress - strain diagram can be used while reflect pulse from the supported end of measuring rod doesn't come to the impacted end. For improving of possibilities this methodology based on the method of characteristics, an algorithm has been developed for calculating the displacements of the impacted end of the measuring rod for a multiply passing strain wave. It is based on the results of the measurements of strains by two gauges which are located near the impacted and supported ends of the measuring rod (figure 2). Accordingly general solution of wave equation velocity $\dot{u}_{z}$ and stress $\sigma_{z}$ are summa of the incident $f$ and reflect $g$ waves which are propagated from impacted to supported ends of the measuring rod correspondingly. The velocity and stress can be found in every point of the measuring rod at any time:

$$
\begin{aligned}
\dot{u}(z, t) & =1 / 2[f(z-c t)+g(z+c t)], \\
\sigma(z, t) & =\rho c / 2[f(z-c t)-g(z+c t)]
\end{aligned}
$$

Functions $f$ and $g$ are defined from an initial and a boundary conditions. In the experiment the boundary conditions of the measuring rod ends of gas-gun stand is not known and it is necessary to define they.

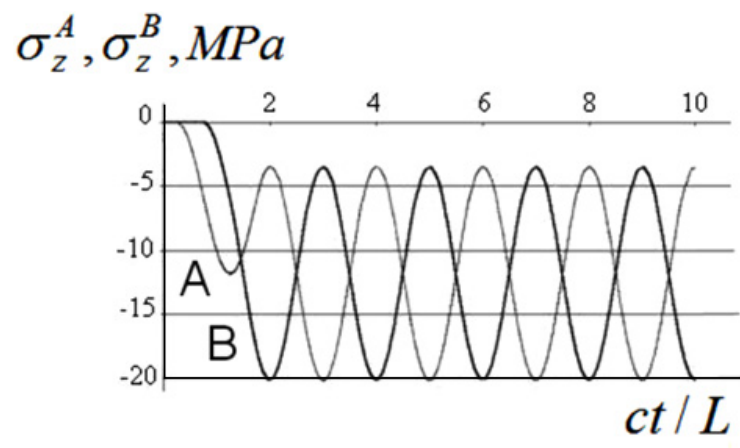

Fig. 2. Axis stresses at location A and B.

Substituting values of the strain gauges coordinate in expression (7) and using Hooke's law, we can get the following expressions:

$$
\begin{aligned}
& E e^{A}(t) \equiv \sigma\left(l^{A}, t\right)=\frac{\rho c}{2}\left[f\left(l^{A}-c t\right)-g\left(l^{A}+c t\right)\right], \\
& E e^{B}(t) \equiv \sigma\left(L-l^{B}, t\right)=\frac{\rho c}{2 E}\left[f\left(L-l^{B}-c t\right)-g\left(L-l^{B}+c t\right)\right],
\end{aligned}
$$

where $e^{A}, e^{B}$ are strains at location $\mathrm{A}$ and $\mathrm{B}, l^{A}$ and $l^{B}$ are the distance from the end of measuring rod to the corresponding gauges, $\mathrm{L}$ is a length of the measuring rod. Using strain pulses $e^{A}(t)$ and $e^{B}(t)$ from the experiment and assuming the function $g=0$ when $c t \leq L$, i.e. while the strain wave is not reflected from the supported end of the measuring rod, it is possible to restore function $f$ and $g$ :

$$
\begin{gathered}
f\left(l^{A}-c t\right)=\frac{2 E}{\rho c} e^{A}(t)+g\left(l^{A}+c t\right), \\
g\left(L-l^{B}+c t\right)=-\frac{2 E}{\rho c} e^{B}(t)+f\left(L-l^{B}-c t\right)
\end{gathered}
$$

Using (7) the boundary conditions can be reconstructed. These are the pulses of velocities and stresses on the ends of the measuring rod.

Using the stress on the impacted end of the measuring rod from this methodology (1)-(6) the dependence of axis stress - axis strain of materials $\sigma_{z} \sim e_{z}$ can be built based on the all process of deformation before stopped moment or rebound of the striker. It allows to compare results of calculation and experiments using the residual size of the specimen.

\section{Testing of the methodology of wave process reconstruction in the measuring rod based on two strain gauges}

For testing of the algorithm for calculation of velocities and stresses numerical modeling of wave process in the measuring rod is performed at one-dimension case. These calculations have been performed by application program package "Dynamic -2" [5]. One ends of the measuring rod is rigidly fixed and other is applied axis velocity by the 


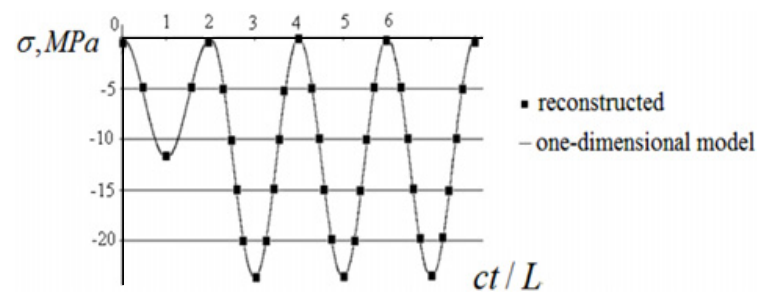

Fig. 3. Stresses on the impacted end of the measuring rod.

following law:

$$
V(t)=\left\{\begin{array}{l}
V_{0} \sin ^{2}\left(\frac{\pi t}{2 T}\right), t<2 T \\
0, t \geq 2 T
\end{array}\right\}
$$

where $T=L / c$ is time while wave is propagating along the measuring rod from the impacted to the supported ends.

On figure 2 axis stresses are shown at location A and B. Distance from the impacted and supported ends to gauges is set to $\mathrm{L} / 4$.

Further, boundary conditions are considered unknown and is restored by reviewed methodology. On figure 3 stresses (one-dimensional model and restored by methodology) on the impacted end of the measuring rod.

Hence, recommend methodology allows to restore boundary condition in the measuring rod for a multiply passing strain wave with accuracy which is defined by measure of inaccuracies of experimental strain measurements.

\section{Constructing dynamic stress - strain diagram for lead based on the data from two strain gauges}

For approbation of developed methodology constructing of dependency of axis stress- axis strain $\sigma_{z} \sim e_{z}$ some experiments of compressing have been realized on gas-gun stand for a multiply passing strain wave. The geometric parameters of the specimen were chosen as follows: the striker radius and length are $R_{1}=3.75 \cdot 10^{-2} \mathrm{~m}$ and $L_{1}=$ $0.24 \mathrm{~m}$, the specimen radius and length are $R_{2}=0.01 \mathrm{~m}$ and $L_{2}=0.02 \mathrm{~m}$, the measuring rod radius and length are $R_{3}=3.75 \cdot 10^{-2} \mathrm{~m}$ and $L_{3}=1.2 \mathrm{~m}$.

The specimen was made of steel with the following elastic characteristics: the bulk compression modulus is $K=1.346 \cdot 10^{5} \mathrm{MPa}$, the shear modulus is $G=8.467$. $10^{4} \mathrm{MPa}$ and the density is $\rho=7.85 \cdot 10^{-2} \mathrm{~kg} / \mathrm{m}^{3}$.

In the experiment strain pulses $e_{z}^{A}, e_{z}^{B}$ are registered by two gauges which are located apart $l^{A}=l^{B}=L_{3} / 4$ from the impacted and supported ends of the measuring rod correspondingly (figure 4). The initial velocity of striker is $16 \mathrm{~m} / \mathrm{s}$.

On figure 5, 6 stresses and velocities on the impacted(marked 1) and the supported(marked 2) ends of the measuring rod are shown which are built by the methodology (7)-(9). On the supported end boundary condition is above free displacement.

On figure 7 the axis velocity of the striker is shown. Time of the striker stopping is about $7 c t / L_{3}$, where $c t / L_{3}$

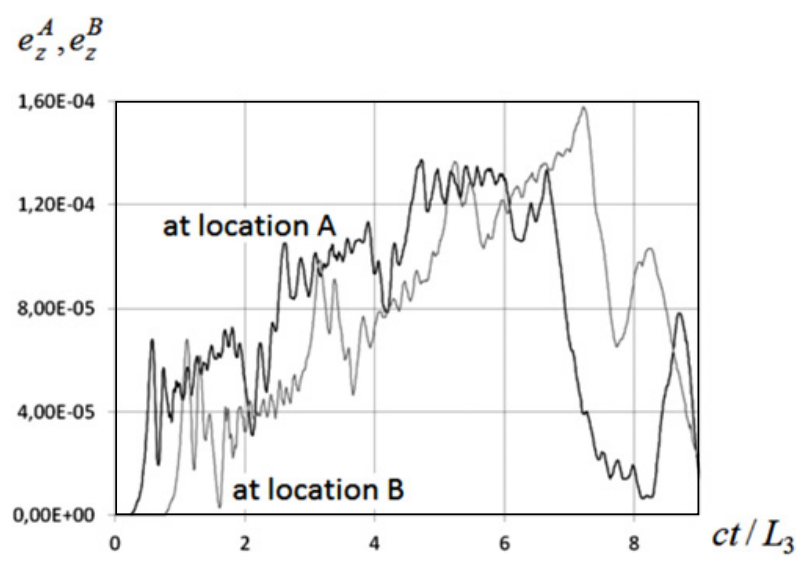

Fig. 4. Strain in gauges at location A and B.

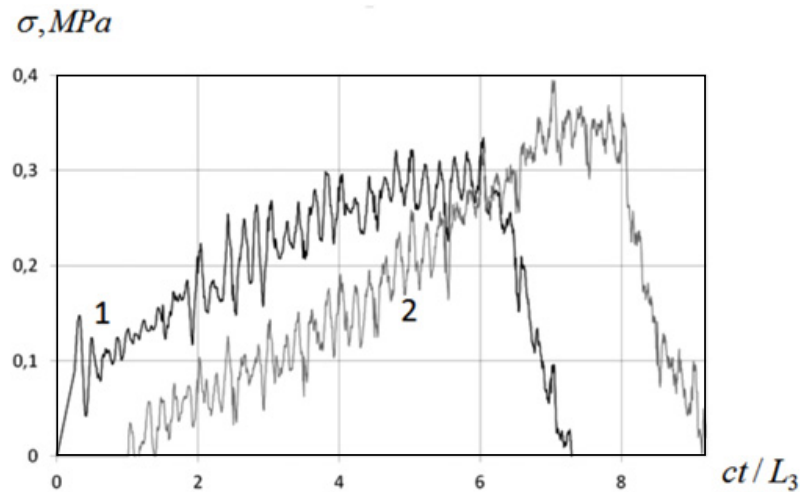

Fig. 5. Stress on the impacted and supported ends of the measuring rod.

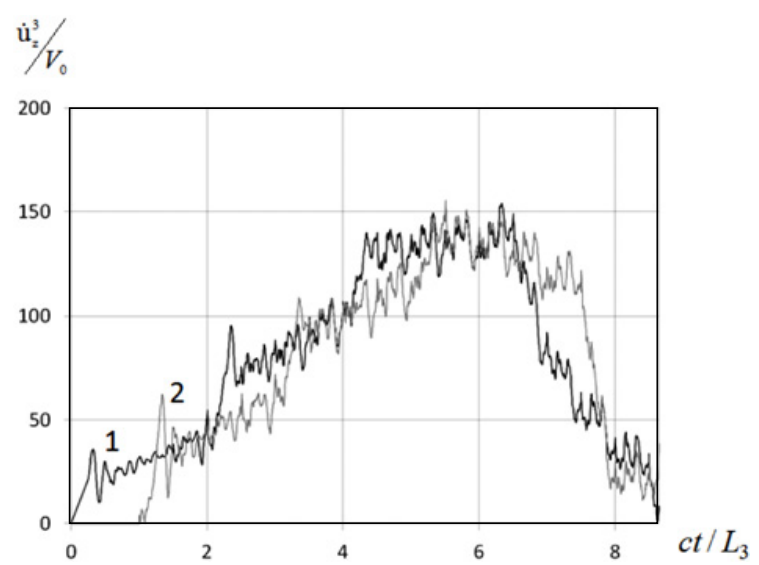

Fig. 6. Velocities on the impacted and supported ends of the measuring rod.

is time while wave is propagating along the measuring rod from the impacted end to the supported one.

On figure 8 the axis displacements of the contact surfaces of the striker (marked 1) and the measuring rod (marked 2) are shown.

Neglect of the measuring rod deformation recasts to error above seven per cent for defining of strain specimen.

On figure 9 nominal (marked 1) and true (marked 2) axis strains in the specimen are shown which are calculated by (5).

On figure 10 dependence axis stress - axis strain (marked 1) which is built by the methodology, it's 
$\dot{u}_{z}^{1} / V_{0}$

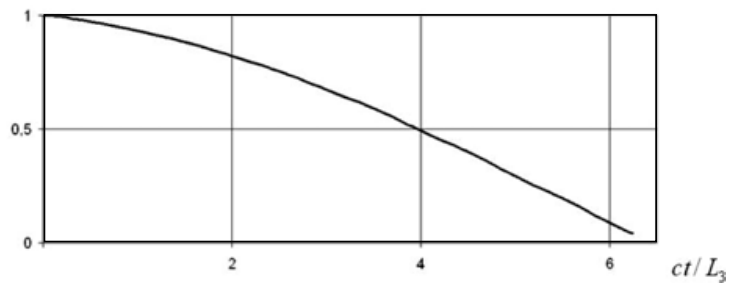

Fig. 7. Axis velocity of the striker.

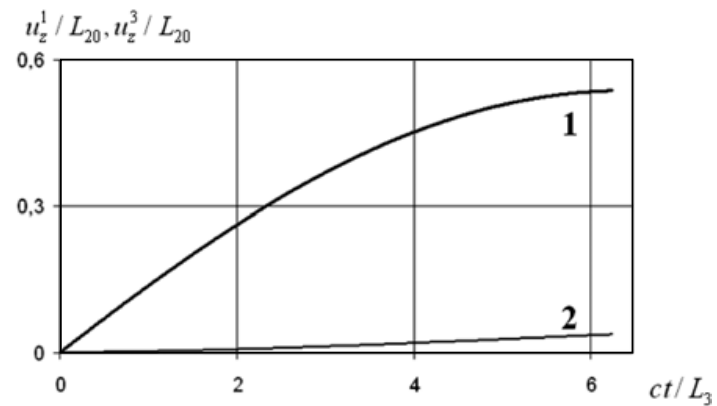

Fig. 8. Axis displacements of the contact surfaces of the striker and measuring rod.

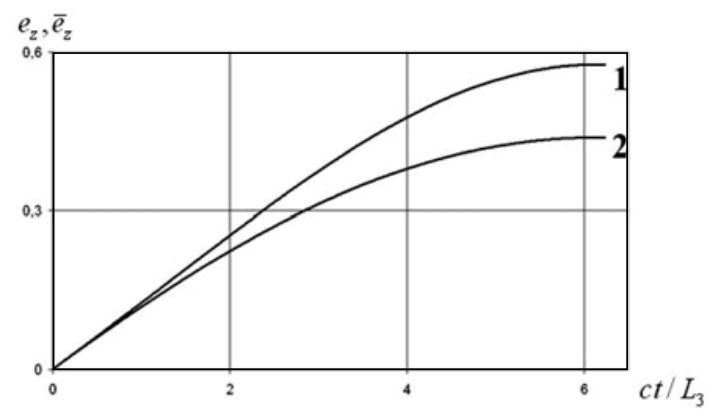

Fig. 9. Nominal and true strains in the specimen.

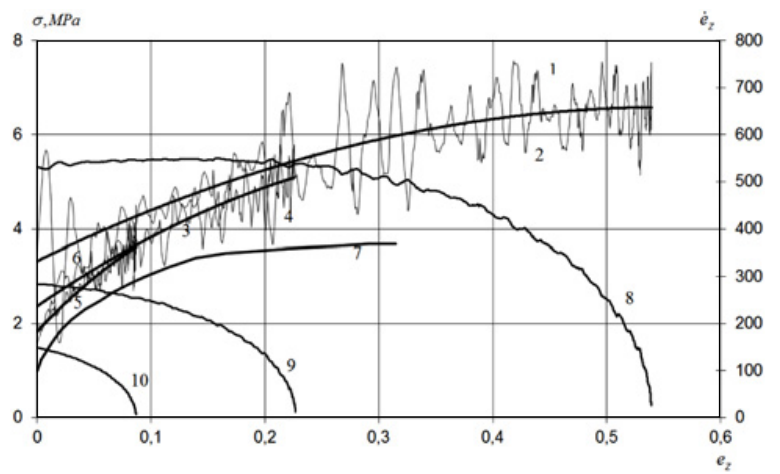

Fig. 10. Stress-strain diagrams of lead.

approximation (marked 2) and dependency axis strain rate (marked 8) from degree of axis strain are shown.

Strain registration by one gauge which is located near the impacted end allows to build dependency axis stress axis strain while the reflected wave will come at location of gauge.

In this experiment axis strain of lead is twenty per cent if one gauge is used. Using system of two gauges allows considering all deformation process.
Also experiments have been done for two velocities of striker: $6.6 \mathrm{~m} / \mathrm{s}, 3.5 \mathrm{~m} / \mathrm{s}$.

On figure 10 dependences axis stress - axis strain for these velocities of the striker (marked 3,5 corresponding velocities $6.6 \mathrm{~m} / \mathrm{s}, 3.5 \mathrm{~m} / \mathrm{s}$ ) which are built by the methodology, their approximation (marked 4,6), dependences axis strain rate(marked 9,10) from degree of axis strain and static strain diagram(marked 7) of lead which is got in Research Institute of Mechanics Of Nizhnij Novgorod State University are shown.

\section{Conclusion}

Developed methodology allows to define the dependence of axis stress from axis strain under compression of specimen by direct impact method for a multiply passing strain wave along the measuring rod. It is very important for building stress - strain diagram of high plasticity materials such as lead at high strains.

\section{Acknowledgements}

The authors thank their colleagues from the Research Institute of Mechanics of the Lobachevskii State University, D.V. Zhegalov, D.A. Kazakov, V.B. Korobov, for their help in conducting the experiments.

The research was financially supported by the Russian Federal Target Program "Scientific and Scientific-Pedagogical Personnel In Innovative Russia for 2009-2013", the Russian Federal President Program fro Supporting Leading Scientific Schools (project No. NSh -2843.2012.8), and the Russian Foundation for Basic Research (project No.11-08-00565-a).

\section{References}

1. F.E.Hauser, J.A. Simmons, J.E. Dorn, Strain rate effects in plastic wave propagation, Response of Metals to High Velocity Deformation edited by P. G. Shewmon and V. F. Zackay, New York: Interscience,P.93-110(1961).

2. J.R.Klepaczko, Advanced experimental techniques in materials testing, New Experimental Methods in Material Dynamics and Impact, Trends in Mechanics in Materials, eds. W.K. Novacki, J.R. Klepaczko, Warsaw, (2001), P. 223-266.

3. U.S. Lindholm, L.M. Yeakley, High strain-rate testing: tension and compression, Exp. Mech. -. 8(1968), N 1., P. 1-9.

4. V.G.Bazhenov, M.S.Baranova, E.V.Pavlenkova, Development and verification of the direct impact method for identifying viscoplastic properties of materials in experiments on a gas dynamic vertical test stand, Problems of resistance and plasticity, N.Novgorod, Stare university, 71(2009), P.184-192.

5. V.G.Bazhenov, S.V.Zefirov, A.V.Kochetkov Application Program Package "Dynamic -2", Problems of strength and plasticity. Research and optimization of constructions. Gorkovsky university(1987), P.4-13. 